\title{
Bildungsrecht: gemeinschaftsrechtliche Maßstäbe
} Ein Rechtsanspruch auf Gleichbehandlung wegen des Geschlechts ist nicht vorgesehen

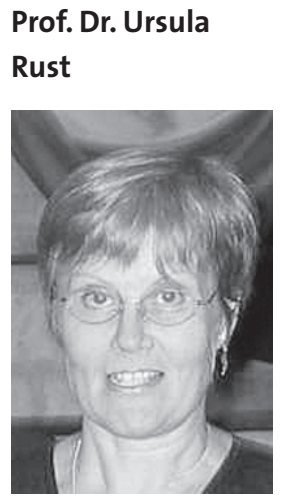

Mitglied der Kommission Recht der sozialen Sicherung, Familienlastenausgleich des djb, Professorin für Gender Law, Arbeitsrecht und öffentliches Recht, Hochschullehrerin an der Universität Bremen
Seit dem Vertrag von Amsterdam von 1997 (konkret: Art. 13 EG) sind Diskriminierungen hinsichtlich der Merkmale Geschlecht, Rasse und ethnische Herkunft, Religion oder Weltanschauung, Behinderung, Alter sowie sexuelle Ausrichtung zu bekämpfen. Auf Art. 13 EG gestützte Richtlinien erfordern Einstimmigkeit im Rat, die bisher mit den Richtlinien 2000/43/EG, 2000/78/EG und 2004/113/EG erreicht wurde. Ergebnis sind Mindeststandards. Sie unterscheiden sich bisher mit Blick auf die jeweiligen Lebens- und Handlungsbereiche und sind für die Merkmale des Art. 13 EG verschieden weit ausgestaltet.

Der Rat berät derzeit über einen weiteren auf Art. 13 EG gestützten Richtlinienvorschlag der Kommission. Bisher regelt die Antirassismusrichtlinie 2000/43/EG vom 29. Juni 2000 zu den Merkmalen Rasse und Ethnie den gemeinschaftsrechtlichen Mindestschutz vor Diskriminierung für den Erwerbsbereich, die allgemeine Bildung, den $\mathrm{Zu}$ gang zu Gütern und Dienstleistungen und zum Sozialschutz. Die Rahmenrichtlinie Richtlinie 2000/78/EG vom 27. November 2000 bestimmt nur für den Erwerbsbereich hinsichtlich der Merkmale Religion oder Weltanschauung, Behinderung,
Alter sowie sexuelle Ausrichtung Mindeststandards. Mindeststandards zum Schutz vor Geschlechterdiskriminierung sind bisher nur für den Zugang zu Gütern und Dienstleistungen mit der Richtlinie 2004/113/EG vom 13. Dezember 2004 begründet worden.

Zur Geschlechtergleichbehandlung hat sich das europäische Recht lange vor dem Amsterdamer Vertrag zum Erwerbsbereich entwickelt. Startpunkt war der seit den Römischen Verträgen im Primärrecht geregelte Grundsatz der Geschlechtergleichbehandlung. 2002 wurde - gestützt auf die Kompetenzgrundlage des Art. 141 Abs. 3 EG - die für die Erwerbstätigkeit geltende Richtlinie 76/207/EG ergänzt. Beide Richtlinien sind durch die Neufassungsrichtlinie 2006/53/EG abgelöst worden. Zu den gesetzlichen Systemen der sozialen Sicherheit sind die gemeinschaftsrechtlichen Standards zum Geschlecht in der bis heute unveränderten Richtlinie 79/7/EWG bestimmt.

Ergebnis ist derzeit, dass die gemeinschaftsrechtlichen Mindeststandards des Diskriminierungsschutzes zu den in Art. 13 genannten Merkmalen bisher hierarchisch ausgestaltet sind, wie Tabelle 1 veranschaulicht.

\begin{tabular}{|l|l|l|l|l|l|l|}
\hline \multicolumn{1}{|c|}{ Gründe } & Rasse/Ethnie & Religion & Behinderung & Alter & $\begin{array}{l}\text { Sexuelle Aus- } \\
\text { richtung }\end{array}$ & Geschlecht \\
\hline $\begin{array}{l}\text { Beschäftigung und } \\
\text { Berufsausbildung }\end{array}$ & $\begin{array}{l}\text { ja }+ \\
\text { Gleichstellungsstelle }\end{array}$ & ja & ja & ja & ja & $\begin{array}{l}\text { ja + } \\
\text { Gleichstellungsstelle }\end{array}$ \\
\hline Allgemeine Bildung & $\begin{array}{l}\text { ja }+ \\
\text { Gleichstellungsstelle }\end{array}$ & nein & nein & nein & nein & nein \\
\hline $\begin{array}{l}\text { Güter und Dienst- } \\
\text { leistungen }\end{array}$ & $\begin{array}{l}\text { ja }+ \\
\text { Gleichstellungsstelle }\end{array}$ & nein & nein & nein & nein & $\begin{array}{l}\text { ja + } \\
\text { Gleichstellungsstelle }\end{array}$ \\
\hline Sozialschutz & $\begin{array}{l}\text { ja }+ \\
\text { Gleichstellungsstelle }\end{array}$ & nein & nein & nein & $\begin{array}{l}\text { teils ja }+ \\
\text { Gleichstellungsstelle }\end{array}$ \\
\hline
\end{tabular}

A Tabelle 1: Geltendes Recht

\begin{tabular}{|l|l|l|l|l|l|l|}
\hline \multicolumn{1}{|c|}{ Gebiet } & Religion & Behinderung & Alter & $\begin{array}{l}\text { Sexuelle Aus- } \\
\text { richtung }\end{array}$ & Geschlecht \\
\hline $\begin{array}{l}\text { Beschäftigung und } \\
\text { Berufsausbildung }\end{array}$ & $\begin{array}{l}\text { ja }+ \\
\text { Gleichstellungsstelle }\end{array}$ & ja & ja & ja & ja & $\begin{array}{l}\text { ja }+ \\
\text { Gleichstellungsstelle }\end{array}$ \\
\hline Allgemeine Bildung & $\begin{array}{l}\text { ja }+ \\
\text { Gleichstellungsstelle }\end{array}$ & ja & ja & ja & ja & nein \\
\hline $\begin{array}{l}\text { Güter und Dienst- } \\
\text { leistungen }\end{array}$ & $\begin{array}{l}\text { ja }+ \\
\text { Gleichstellungsstelle }\end{array}$ & ja & ja & ja & ja & ja + \\
\hline Sozialschutz & $\begin{array}{l}\text { ja }+ \\
\text { Gleichstellungsstelle }\end{array}$ & jastellungsstelle \\
\hline
\end{tabular}

A Tabelle 2: Vorgeschlagene Änderungen 
Dies soll sich jetzt ändern: die allgemeine Bildung soll hinsichtlich aller Merkmale vor Diskriminierung geschützt sein Ausnahme: das Geschlecht. Im Juli 2008 hat die Kommission den Vorschlag für eine Richtlinie des Rates zur Anwendung des Grundsatzes der Gleichbehandlung ungeachtet der Religion oder der Weltanschauung, einer Behinderung, des Alters oder der sexuellen Ausrichtung vorgelegt. ${ }^{1}$ Mit dem Erlass einer vierten auf Art. 13 gestützten Richtlinie würde das Mindestschutzniveau für alle in Art. 13 genannten Merkmale bis auf das Geschlecht angeglichen werden. Zum Geschlecht würde die Lücke des Diskriminierungsschutzes für die Sozialleistungen und die allgemeine Bildung zum Geschlecht weiterhin bestehen bleiben. Die vorgeschlagenen Änderungen zeigt Tabelle 2.

Im europäischen Jahr der Chancengleichheit 2007 sind von der EG-Kommission zunächst interessierte Kreise wie die Geschäftswelt, die Sozialpartner sowie - breiter angelegt mit einer Online-Konsultation - auch die Öffentlichkeit zu möglichen Vorschlägen befragt worden.

Der Vorschlag der Kommission entspricht auch einem vom Europäischen Parlament geäußerten Wunsch. ${ }^{2}$ Dort und im Rat wird der Richtlinienentwurf derzeit beraten. ${ }^{3}$

Die Regelungslücke hinsichtlich der Sozialleistungen und zur allgemeinen Bildung würde dem Grundsatz des Art. 3 Abs. 2 EG widersprechen, bei allen Tätigkeiten der Gemeinschaft darauf hinzuwirken, Ungleichheiten zu beseitigen und die
Gleichstellung von Frauen und Männern zu fördern, der ebenfalls seit dem Vertrag von Amsterdam gilt. Zum Tragen kommen die zum Gleichbehandlungsrecht getrennten Diskurse der beteiligten Nichtregierungsorganisationen. So spiegeln sich auch u.a. die für die Bekämpfung von Diskriminierung und der Gleichstellung der Geschlechter getrennten Zuständigkeiten bei der EG-Kommission und beim Europäischen Parlament zwischen dem Ausschuss für Bürgerliche Freiheiten, Justiz und Inneres (LIBE) und für Rechte der Frau und Gleichstellung der Geschlechter (FEMM) mit offensichtlich unterschiedlichen Durchsetzungschancen wider. Anfang 2009 hat der zuständige Ausschuss des Europäischen Parlaments gegenüber der Kommission die Erwartung geäußert, den Schutz gegen Diskriminierung wegen des Geschlechts auf das Niveau zu bringen, das für die anderen in Art. 13 EG genannten Merkmale gilt. Die bestehende Hierarchie ist zu beseitigen. Hierzu werden Vorschläge der Kommission für 2010 erwartet. $^{4}$

\section{Umsetzung des AGG in den Betrieben: Außer Spesen nichts gewesen?}

Das Allgemeine Gleichbehandlungsgesetz (AGG) ist seit 14 . August 2006 in Kraft, also seit gut zweieinhalb Jahren. Unsere Unternehmensbefragung wollte untersuchen, ob und wie das AGG nach zwei Jahren umgesetzt wurde und welche Veränderungen es für die betriebliche Praxis gebracht hat. Zwischen Januar und Mai 2008 führte das Hamburger AGG-Projekt ${ }^{1}$ im Großraum Hamburg 52 leitfadengestützte Experteninterviews mit einer Dauer zwischen 40 Minuten und zwei Stunden. Persönlich befragt wurden 52 Personen aus 41 Unternehmen der Privatwirtschaft: 36 Vertreter/innen aus den Personalabteilungen bzw. der Geschäftsleitung, acht Mitglieder des Betriebsrats und acht Beauftragte für Gleichstellung, für Behinderte oder sonstige Minoritäten. Die Befragung war nicht repräsentativ angelegt, verteilte sich aber recht gleichmäßig auf die unterschiedlichen Unternehmenstypen und Branchen. ${ }^{2}$ Ihre Ergebnisse sind damit zumindest für städtische Ballungsräume durchaus aussagekräftig.

\section{Die Beurteilung des AGG bei seiner Einführung}

Die deutsche Wirtschaft lehnte die neuen rechtlichen Vorgaben 2006 mehrheitlich ab und prophezeite Klagewellen von abgelehnten Bewerber/innen und hohe Umsetzungskosten. In einem „Stimmungsbild“ unter Personalmanagern $2006^{3}$ fanden sich Aussagen wie: „Das AGG ist ein Hammergesetz, das uns in vielen Punkten fordern wird.“ „Das AGG lädt dazu ein, es schamlos auszunutzen.“ „Vor allem in der Anfangszeit wird es Klagen hageln.“ „Alles in allem sehen wir zu viel Regelungsund Überwachungsbedarf.“

1 Projektleitung: Sibylle Raasch und Daniela Rastetter, Projektmitarbeiterinnen: Nina Bielau, Diana Fazari, Ursula Kisse, Katarzyna Najlepsza, Emma Patrignani, Tinka Rieckhoff, Regine Starck, Guanayu Hou.

2 Vgl. ausführlicher Ergebnisbericht Raasch, S.; Rastetter, D.: Die Anwendung des AGG in der betrieblichen Praxis, Hamburg Januar 2009.

3 Personalmagazin, Titelthema: Das AGG im Urteil der Personalmanager, 7/2006, S. 28-29.

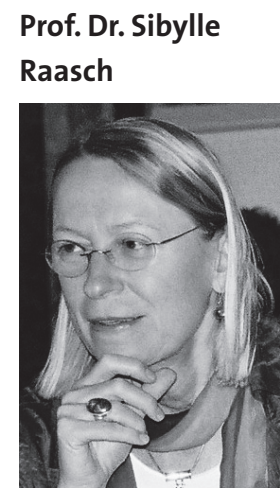

Vorsitzende der Kommission Arbeits-, Gleichstellungs- und Wirtschaftsrecht des djb, Professur für Öffentliches Recht und Gender Studies, Fachbereich Sozialökonomie, Universität Hamburg 